\title{
Clinical audit in nuclear medicine
}

\author{
Siroos Mirzaei • Lorenzo Maffioli • Andrew Hilson
}

Published online: 25 September 2010

(C) Springer-Verlag 2010

Nuclear medicine is one of the most dynamic fields in medicine. It is defined in the new Accreditation Council for Graduate Medical Education (ACGME) program requirements as "Nuclear medicine is the medical specialty that uses the tracer principle, most often with radiopharmaceuticals, to evaluate molecular, metabolic, physiologic and pathologic conditions of the body for the purposes of diagnosis, therapy and research" [1]. Training in nuclear medicine, which comprises a period of 4 to 6 years, has undergone major revisions since its start. The field of investigations has also undergone a more rapid transformation than many other specialties in many areas from instrumentation to radiopharmacy. The radiopharmaceuticals available are fewer but are also more specific than in earlier years, and many of the common procedures performed previously are no longer offered in modern routine clinical nuclear medicine. Many earlier studies have become outdated, such as renal studies with ${ }^{131}$ I-orthoio-

\section{S. Mirzaei}

Wilhelminenspital, Institute of Nuclear Medicine with PET-center,

Vienna, Austria

L. Maffioli $(\square)$

Ospedale Civile di Legnano, Direttore U.O. di Medicina Nucleare,

Via Candiani, 2,

20025 Legnano, Italy

e-mail: lorenzo.maffioli@ao-legnano.it

A. Hilson

Royal Free Hospital,

London, UK

S. Mirzaei $\cdot$ L. Maffioli $\cdot$ A. Hilson

Committee for Accreditation of Nuclear Medicine Departments, UEMS/EBNM,

Wien, Austria dohippurate, ventilation studies with ${ }^{133} \mathrm{Xe}$ or pancreas imaging with ${ }^{75} \mathrm{Se}$-selenomethionine. Major new procedures and materials that have been incorporated into nuclear medicine in the last three decades include myocardial perfusion imaging with technetium-based agents, thyroid imaging with ${ }^{123}$ I, hepatobiliary imaging, aerosol ventilation studies, labelled white blood cells, renal scans, somatostatin analogues, $\beta$-CIT, meta-iodobenzylguanidine, and parathyroid imaging. The fastest growing new modality is positron emission tomography (PET), especially ${ }^{18} \mathrm{~F}$ FDG PET. With hybrid SPECT/CT and PET/CT devices, which create coregistered anatomical and functional images, fusion studies have become more accurate than examinations with stand-alone scanners. A significant number of functional imaging procedures have become a part of the routine clinical work-up for different pathologies. However, some have recently been challenged, as was the case for pulmonary scintigraphy, where multislice CT has replaced the pulmonary perfusion scan in some centres to a large extent. It has to be emphasized that this replacement depends strongly on the experience and quality of the nuclear medicine departments.

Implementing clinical audit in order to improve the quality of the outcomes of nuclear medicine examinations may lead to the decision to keep pulmonary perfusion scintigraphy in the diagnostic tree as a noninvasive modality with exposure of the patient to much less radiation compared to multislice CT. This could be investigated by an "audit patient dose". A major concept introduced by EC directive 97/43/EURATOM [2] is Clinical Audit, which the member states are required to implement in accordance with national procedures. Clinical Audit is defined as a tool to improve the quality of patient care, experience and outcome through formal review of systems, pathways and outcomes of care against defined standards, and the implementation of changes based on the 
results [3]. In other words, it compares what we are doing to what should be done by judging our present clinical practice in relation to the national or international standards for the given situation [4]. Comparing to research, audit has its roots in management ensuring things are done right, while research has its roots in science and academia discovering the right thing to do [5].

So far, only Finland has implemented the EC regulation [2] by national legislation. The Radiation and Nuclear Safety Authority in Finland supervises clinical audits which are carried out every fifth year [6]. A conference was held in Tampere in 2003 to assess the status of Clinical Audit in Europe. This was followed by the establishment of an EU working party on the topic which reported in 2008. Its report was published by the EU in 2009 as European Commission Guidelines On Clinical Audit For Medical Radiological Practices "Diagnostic Radiology, Nuclear Medicine And Radiotherapy" [7]. At the same time, the IAEA has produced a publication on Clinical Audit "Quality Management Audits In Nuclear Medicine Practices" [8] - which is necessary for an audit. The European Society of Radiology has published a paper with the necessary steps for an audit in some detail with regard to structure, process and outcome. The paper concludes that a professionally conducted audit is a powerful tool to improve patient care [3]. A regular clinical audit is a formal surveillance of any work steps in a department with the aim of maximizing the safety and minimizing the possible harm to the individuals involved. In an ideal world, a Clinical Audit would examine the quality of the clinical practice of a department by reference to the accuracy of the outcomes. For instance, "In what proportion of patients in whom bone metastases are diagnosed are they really present?" However, this is almost impossible in the real world. It is therefore necessary to use auditing of processes, on the assumption that a department which has good processes in place is more likely to have good outcomes.

The Committee on Accreditation of Nuclear Medicine Departments of the EBNM has required that any Department seeking accreditation should have a Quality Management System in place, and one option for this is now the introduction and use of a Clinical Audit process validated by the National Society. The recommendation of the EU working party is that this should be based on the IAEA process. Part of the Clinical Audit process is that there should be written protocols traceable to National or International guidelines. However, it is also necessary to consider the indications for procedures - "Justification". For example, it has been shown that MRI is more sensitive than ${ }^{99 \mathrm{~m}} \mathrm{Tc}$ MIBI scintigraphy for assessing the involvement of the spine in multiple myeloma [9]. In other words, where MRI is applicable the primary use of the MIBI-scan for staging of these patients is less justified, but in patients with metallic implants or renal impairment the decision should be the reverse. Similarly, in a young patient with low likelihood of coronary artery disease the use of myocardial perfusion imaging is not justified, unless the treadmill test is positive. These issues also overlap with the ethical aspects of using ionizing radiation [10]. Regarding hybrid devices it would be necessary particularly to evaluate the justification for the use of diagnostic CT and the administration of contrast medium, i.e. in the follow-up of malignant lymphoma where a low-dose CT scan would in most cases be sufficient. However, it should be emphasized that an audit will not always reveal the justification for one specific procedure and the effect on patient management.

For good practice, it is important to consider all these issues. A formal Clinical Audit process will ensure that adequate systems are in place, but it is also important to consider some form of outcome audit - which is much more challenging. This might include some form of peer review of reports, but should also attempt to consider the utility of the nuclear medicine process in the overall patient care.

\section{References}

1. Accreditation Council for Graduate Medical Education. ACGME program requirements for graduate medical education in nuclear medicine. http://www.acgme.org/acWebsite/downloads/ RRC_progReq/200_nuclear_medicine_07012011.pdf Accessed 4 Sep 2010

2. Council Directive (EC) 97/43/Euratom. http://ec.europa.eu/energy/ nuclear/radioprotection/doc/legislation/9743_en.pdf. Accessed 4 Sep 2010

3. European Society of Radiology. Clinical audit-ESR perspective. Insights Imaging 2010;1:21-6.

4. Prince C, Ragesh K, Nisha A. Audit in clinical practice. Indian J Otolaryngol Head Neck Surg 2009;61:109-11.

5. Cave E, Nichols C. Clinical audit and reform of the UK research ethics review system. Theor Med Bioeth 2007;28:181-203.

6. Proceedings of the International Symposium on Practical Implementation of Clinical Audit for Exposure to Radiation in Medical Practices. Tampere, Finland, 24-27 May 2003. http://www. clinicalaudit.net/img/Proceedings2003.pdf. Accessed 4 Sep 2010

7. European Commission. Radiation Protection No. 159. Guidelines on Clinical Audit for Medical Radiological Practices (Diagnostic Radiology, Nuclear Medicine and Radiotherapy. http://ec.europa. eu/energy/nuclear/radiation_protection/doc/publication/159.pdf. Accessed 4 Sep 2010

8. International Atomic Energy Agency. Quality Management Audits in Nuclear Medicine Practices. http://www-pub.iaea.org/MTCD/ publications/PDF/Pub1371_web.pdf. Accessed 4 Sep 2010

9. Mirzaei S, Filipits M, Keck A, Bergmayer W, Knoll P, Koehn H, et al. Comparison of technetium-99m-MIBI imaging with MRI for detection of spine involvement in patients with multiple myeloma. BMC Nucl Med 2003;3:2.

10. Corrao S, Arnone G, Arnone S, Baldari S. Medical ethics, clinical research, and special aspects in nuclear medicine. Q J Nucl Med Mol Imaging 2004;48:175-80. 